\title{
Polycaprolactone - new insight into classic material
}

\author{
Karol Leluk ${ }^{1, *}$, Karolina Sobczyk ${ }^{1}$, and Maciej Borowczak ${ }^{1}$ \\ ${ }^{1}$ Wrocław University of Science and Technology, Department of Environmental Engineering, Poland
}

\begin{abstract}
An investigation on electrospinning of polycaprolactone polymer solution was provided in different process conditions. Although, in all attempts a structure of highly surface-to-mass ratio was achieved, alternation of spinning parameters resulted in products of different geometry. This opens a new way of controlled processing highly porous materials with broad field of application.
\end{abstract}

\section{Introduction}

Polycaprolactone (PCL) is a representative of quite rare group of polymer plastics: originating from fossil fuels (not renewable) on the one hand but being susceptible to degradation processes carried out by microorganisms on the other [1]. Of course, there exist also polymers of the same nature as PCL but this particular plastic is widespread among them. Polycaprolactone belongs to a group of aliphatic polyester polymers and is synthesized in a ring opening polymerization from $\varepsilon$-caprolactone [2]. Due to its specific properties it has been applied in many branches: from implantation technology (scaffolds, drug controlled releasing systems) to vehicle modelling, packaging and artistic plastic products (hobbyists) [3-6]. To name only few of these properties: -low processing temperature (around $58^{\circ} \mathrm{C}$ ), -stability in water environment, -wide range, easy controlled during production stage, mean molecular ratio (related to chain length), -slow degradation rate [7-10]. Contrary to that, PCL's poor mechanical properties and low melting temperature vastly restrict the field of its broad application. That is why even reinforced PCL composites do not reveal a promising materials in the field of load bearing materials. The last thing, but not of least importance, is its high unit price which according to the European market currently oscillates around $15 \mathrm{EUR} / \mathrm{kg}$ (VAT excl.). Why then focus on this niche polymer, which price makes it a remarkably unattractive material?

Starting from economical aspect, the price is strongly affected by material's production rate. According to the research and forecast published by Persistence Market Research [11] European production of PCL is going to dominate reaching 39 of global shares in 2021. The market of PCL (now estimated ca. $115 \mathrm{mln}$ USD) is going to double its value till 2021. That should influence the unit price causing its subsequent decrease. The next issue is ease of material processing: stable in water environment, biodegradable (no processing waste and

\footnotetext{
*Corresponding author: karol.leluk@pwr.edu.pl
} 
end-life product problems). Also its application in electrospinning technology is kind of plug and play: solutions are easy to prepare in a wide range of concentration.

Producing structures with high volume-to-mass ratio has been significant not only from scientific point of view. These are willingly utilised in a transportation sector (lowering mass of a carriage increases fuel consumption savings), remediation and filtration technologies (gas/liquid absorption and adsorption processes), packaging application (active or smart packaging - subsequent release of active factor) and not to forget every-day issues like subsequent odour release. As on the market exist technologies like autoclaving, physical or chemical extrusion foaming, allowing to produce porous, micro- and meso- porous units [12], electrospinning is a technology that is expected to put one step further: creation nano-porous systems with controlled size, shape and porosity factor with negligible influence of processing technology on the material [13]. Creation of different forms in shape (fibers, dots, beds) allows to produce open-work system where the gaps among solid product are not oval-like in shape any more as it is observed in classically manufactured foams.

According to the basics of electrospinning theory, a solution that is capable to be electrospun should have concentration high enough to ensure surface tension and viscosity forces on the sufficient level to polarize the drop and create Taylor cone. Usually the concentration ranges from $5-15 \%(\mathrm{w} / \mathrm{w})$ as the lowest to start the process. Obviously the value depends on polymer internal properties: its nature (linear, branched, block) and carbon main chain length (mean molecular weight). Investigations revealed that the amount of polymer per solvent volume can lead to different forms of end product: low concentrations produce spheres while high end up in fibered systems [14].

The aim of this paper is to describe influence of different concentration and process parameters on the final product as well investigated the influence of the process on the polymer stability.

\section{Experimental section}

\subsection{Materials and sample preparation}

PCL used in the study was delivered by Perstorp, UK (Solvay) under the commercial name $\mathrm{CAPA}^{\circledR} 6400$. According to the manufacturer's note, its mean molecular weight is $37000 \mathrm{~g} / \mathrm{mol}$. Some of its physical properties are listed in the table 1 . Polycaprolactone pellets were dried in the oven before solution preparation. Chloroform (99.5\% pure) was supported by Chempur Poland and used without further purification.

Table 1. CAPA ${ }^{\circledR} 6400$ physical parameters.

\begin{tabular}{|c|c|}
\hline Parameter & Value \\
\hline Melting temperature, ${ }^{\circ} \mathrm{C}$ & $58-60$ \\
\hline Water content, $\%$ & $<1.0$ \\
\hline Elongation at break, $\%$ & 660 \\
\hline MFR $\left(2.16 \mathrm{~kg}, 160^{\circ} \mathrm{C}\right), \mathrm{g} / 10 \mathrm{~min}$ & 40 \\
\hline $\mathrm{OH}$ value, $\mathrm{mg} \mathrm{KOH} / \mathrm{g}$ & 4 \\
\hline
\end{tabular}


Six solutions (of concentration 10\%-35\%, step 5\%) were prepared as follows: demanded quantity of solid sample was weighed on the laboratory scale, put into the vessel and calculated amount of solvent was poured into. Mixing was performed in tightly closed container on the magnetic stirrer until complete dissolution of PCL pellets. No heat was applied to the system.

\subsection{Electrospinning process}

Electrospinning was performed on Fluidnatek LE-10 by Bioinicia (Valencia, Spain) with flat collector. Process conditions, as they slightly differ in all experiments, are mentioned in the further section. Polymer solution was poured into the syringe just before the process to avoid an excessive solvent evaporation.

\subsection{SEM investigation}

Scanning electron microscope was used to investigate the morphology of the process products. It was carried on VEGA Tescan 3 , gun voltage $5.0 \mathrm{kV}$, magnification rate is mentioned on the respective pictures and text. Samples were gold sputtered (Kressington 108 sputter coater) for $60 \mathrm{~s}$ and $40 \mathrm{~mA}$ current.

\subsection{FT-IR (ATR) measurement}

The infrared spectra was obtained for material before dissolution and after electrospinning process. Investigation was performed in the wavenumber range $600-4000 \mathrm{~cm}^{-1}$ using Bruker Tensor 37 apparatus and Opus software. The samples of electrospun materials were placed in the apparatus without any further processing. PCL pellets (solid material before solving) were powdered in grinded. Prior to that, according to the pellets' toughness, the materials were immersed in a liquid nitrogen for about 3 minutes. The powdered polymer sample was then placed into the apparatus measuring cell and the spectra was recorded. Baseline correction, normalization, spectrum smoothening and any further necessary calculations (like peak picking) were done within the measuring procedure time-length. No spectra processing was done after the measurement.

\section{Results and discussion}

It's convenient to start results analysis from infrared investigation which was carried out to check if the electrospinning process affects polymer material or its influence is minor. Plot presented on the figure 1 reveals data collected from raw material (neat PCL) in comparison with two samples picked up from the internal (PCL inner) and external (PCL outer) part of the product. As it is clearly seen, all three spectra are overlapping and can be compared in qualitative (signals position) and quantitative way (signals intensity). For PCL some of the characteristic bands [15]:

- $\quad$ asymmetric $-\mathrm{CH}_{2}$ mode at $2940 \mathrm{~cm}^{-1}$ (pair with $2860 \mathrm{~cm}^{-1}$ )

- $\quad-\mathrm{C}=\mathrm{O}$ stretching mode at $1724 \mathrm{~cm}^{-1}, 1748 \mathrm{~cm}^{-1}$

- $\quad$ C-O stretching mode at $1293 \mathrm{~cm}^{-1}$ and $1149 \mathrm{~cm}^{-1}$

- $\quad$ C-O-C- assymetric stretching mode at $1166 \mathrm{~cm}^{-1}, 1182 \mathrm{~cm}^{-1}$ 


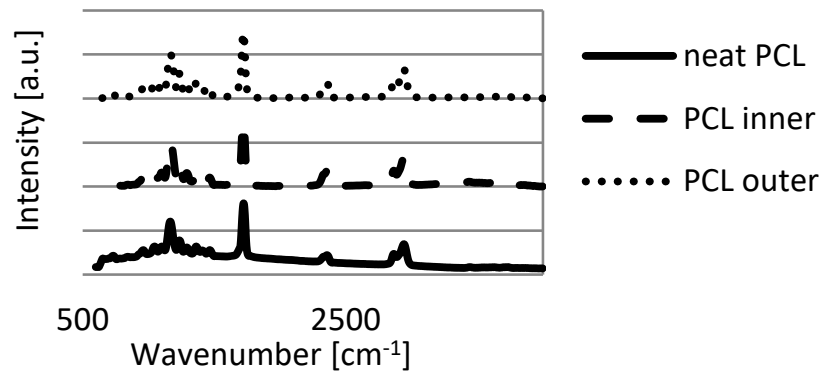

Fig. 1. FT-IR spectra of polycaprolactone before and after electrospinning.

Respective signals were picked up and put into the table 2 for more clear representation. Even brief analysis leads to the conclusion that signals differ only slightly either in their position and intensity. Samples denoted as inner PCL and outer PCL overlap in good agreement with neat polymer what is a satisfactory evidence showing that electrospinning has no significant effect on the chemical and spatial structure.

Table 2. Peak picking analysis for PCL.

\begin{tabular}{|c|c|c|c|}
\hline Sample name & Wavenumber $\left[\mathbf{c m}^{-\mathbf{1}}\right]$ & Intensity (Abs) & Width $\left[\mathbf{c m}^{-\mathbf{1}}\right]$ \\
\hline neat PCL & 2943 & 0.7 & 58.6 \\
\hline & 1724 & 1.6 & 31.4 \\
\hline & 1166 & 1.2 & 48.6 \\
\hline & & & 66.9 \\
\hline PCL inner & 2942 & 0.6 & 59.1 \\
\hline & 2358 & 0.4 & 32.9 \\
\hline & 1722 & 1.5 & 27.0 \\
\hline & 1238 & 0.5 & 47.6 \\
\hline & 1169 & 1.0 & 66.4 \\
\hline & & & 59.0 \\
\hline PCL outer & 2942 & 0.7 & 32.9 \\
\hline & 2358 & 0.3 & 27.0 \\
\hline & 1722 & 1.5 & 48.2 \\
\hline & 1238 & 0.6 & \\
\hline & 1168 & 1.0 & \\
\hline
\end{tabular}

Main investigation of the paper is focused on fabrication of porous PCL systems. It is a common knowledge that altering concentration of polymer solution leads to products that differ in shape. At highly concentrated solutions, commonly observed are fibered products (with fiber diameter of several micrometers) whereas low concentrated ones lead to spheric product.

Figure 2 represents micrographs obtained using scanning electron microscope mainly at magnification rate 500-1000 x for six different concentrations. In table 3 a list of all process parameters related to particular samples are listed together with used below sample abbreviation.

Concentrations up to $20 \%$ result in spinning of sphere-shape, regular structures. In samples S1-S3 mean diameter of spheres vary in range $25-35 \mu \mathrm{m}$ irrespective to change in concentration. Visible minor, residual fibers (with diameter $200-300 \mathrm{~nm}$ ) are stick to the spheres surface. 
Table 3. Electrospinning process parameters for subsequent PCL samples.

\begin{tabular}{|c|c|c|c|c|}
\hline $\begin{array}{c}\text { Sample name } \\
\text { abbreviation }\end{array}$ & $\begin{array}{c}\text { Concentration } \\
{[\mathbf{\%}]}\end{array}$ & $\begin{array}{c}\text { Collector } \\
\text { distance } \\
{[\mathbf{c m}]}\end{array}$ & $\begin{array}{c}\text { Nozzle } \\
\text { voltage }[\mathbf{k V}]\end{array}$ & $\begin{array}{c}\text { Liquid flow } \\
{[\mathbf{m l} / \mathbf{h}]}\end{array}$ \\
\hline S1 & 10 & 20 & 13.3 & 1.0 \\
\hline S2 & 15 & 22 & 5.6 & 1.0 \\
\hline S3 & 20 & 22 & 5.8 & 0.8 \\
\hline S4 & 25 & 22 & 5.6 & 0.8 \\
\hline S5 & 30 & 22 & 6.6 & 1.0 \\
\hline S6 & 35 & 22 & 5.8 & 0.8 \\
\hline S7 & 35 & 27 & 5.8 & 0.8 \\
\hline
\end{tabular}

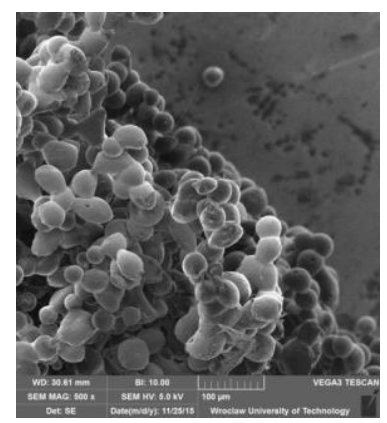

$\mathrm{S} 1-500 \mathrm{x}$

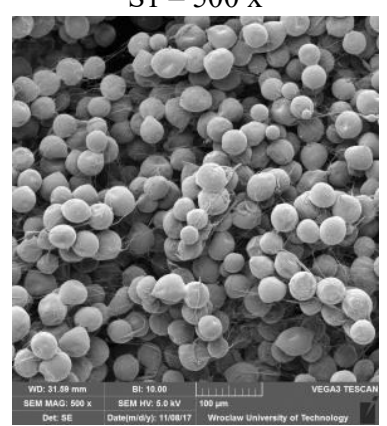

$\mathrm{S} 3-500 \mathrm{x}$

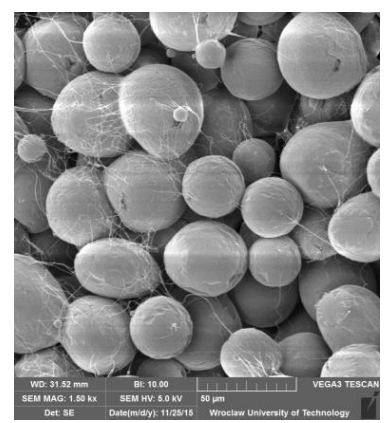

$\mathrm{S} 1-1500 \mathrm{x}$

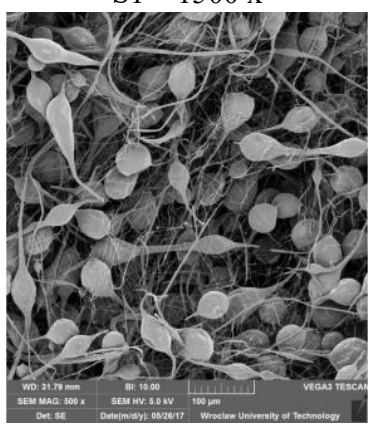

$\mathrm{S} 4-500 \mathrm{x}$

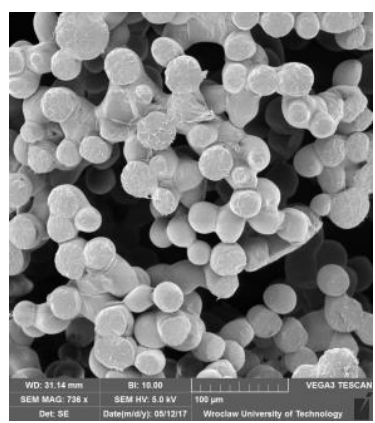

$\mathrm{S} 2-800 \mathrm{x}$

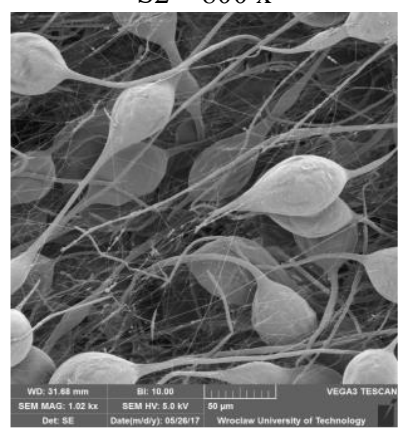

S5 $-1000 \mathrm{x}$

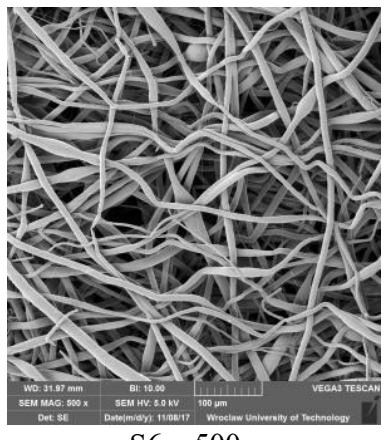

S6 - $500 \mathrm{x}$

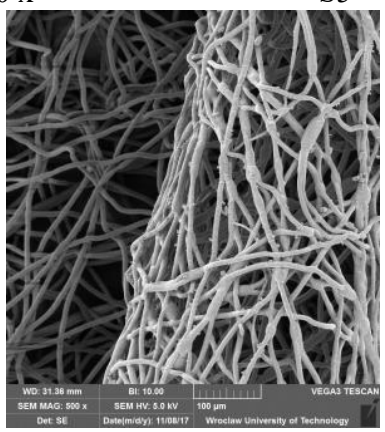

S7 $-500 x$

Fig. 2. SEM micrographs for all investigated samples. Magnification rate shown next to sample abbreviation. 
Starting from solution with PCL concentration $25 \%$ (S4) one can easily observe tendency to fibers formation. While in S4 and S5 samples strong polarization of spheres resulted in beaded fiber formation, the last two represent fibers with minor evidence of beads. Secondly, the amount of residual fibers increased in S4 and S5 samples reaching its top value, until PCL concentration reached 35\% in S6 sample where presence of residual fibers can be neglected. What is also important, together with increasing concertation the mean diameter of fibers reveals decreasing tendency: $40 \mu \mathrm{m}$ (S6), $15 \mu \mathrm{m}$ (S7).

\section{Conclusions}

- Electrospinning process does not affect chemical structure nor sterical orientation of investigated polycaprolactone;

- increasing polymer's concentration results in creation structures with pronounced fibrous character;

- the threshold concentration (for fibre formation) was found to be $35 \%(\mathrm{w} / \mathrm{w})$;

- all non-fibrous structures reveal symmetric shape;

- above investigation proved that electrospinning is a versatile technique allowing to produce structures of high volume-to mass ratio but with differing geometrical parameters (spheres, spheroids, beaded fibres, fibres).

\section{References}

1. L. Averous, J. Macromol. Sci. Polymer Rev. 44, 231 (2004)

2. N. Susperregui, D. Delcroix, B. Martin-Vaca, D. Bourissou, L. Maron, J. Org. Chem. 75, 6581 (2010)

3. L. Atanasoska, J.S. Stinson, Ch. Deng, D. Boismier, J. Weber, T. Scheuemann, U.S. Patent, No. 2011/0022158 A1 (2011)

4. C.G. Pitt, F.J. Chasaldo, J.M. Hibionada, D.M. Klina, A. Schindler, Appl. Polym. Sci. 26, 3779 (1981)

5. Ph. Dubois, C. Jacobs, R. Jerome, Ph. Teyssie, Macromolecules 24, 2266 (1991)

6. G. Davis, J.H. Song, Ind. Crop. Prod. 23, 147 (2006)

7. D.R. Chen, J.Z. Bei, S.G. Wang, Polym. Degrad. Stabil. 67, 455 (2000)

8. F. Lefebvre, C. David, C. Van der Wauven, Polym. Degrad. Stabil. 45, 347 (1994)

9. C. Eldsäter, B. Erlandsson, R. Renstad, A.-C. Albertsson, S. Karlsson, Polymer 41, 1297 (2000)

10. S. Ponsart, J. Coudane, B. Saulnier, Jean-Louis Morgat, M. Vert, Biomacrom. 2, $373(2001)$

11. https://www.newswire.com/news/global-polycaprolactone-market-forecast-to-2021europe-is-expected-to-dominate - available on 25-01-2018

12. L. Shau-Tarng, Polymeric Foams: Innovations in Processes, Technologies, and Products, 36 (2016)

13. Ch.J. Angammana, S.H. Jayaram, Part. Sci. Technol. 34, 72 (2016)

14. Z. Li, C. Wang, One-Dimensional nanostructures. Electrospinning Technique and Unique Nanofibers, 15 (2013)

15. E.M. Abdelrazek, A.M. Hezma, A. El-khodary, A.M. Elzaya, Egypt. J. Basics Appl. Sci. 3, 10 (2016) 